\title{
OPEN Scaffold-based 3D cellular models mimicking the heterogeneity of osteosarcoma stem cell niche
}

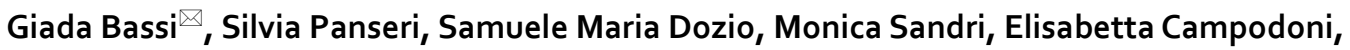 \\ Massimiliano Dapporto, Simone Sprio, Anna Tampieri \& Monica Montesi ${ }^{\bowtie}$
}

The failure of the osteosarcoma conventional therapies leads to the growing need for novel therapeutic strategies. The lack of specificity for the Cancer Stem Cells (CSCs) population has been recently identified as the main limitation in the current therapies. Moreover, the traditional twodimensional (2D) in vitro models, employed in the drug testing and screening as well as in the study of cell and molecular biology, are affected by a poor in vitro-in vivo translation ability. To overcome these limitations, this work provides two tumour engineering approaches as new tools to address osteosarcoma and improve therapy outcomes. In detail, two different hydroxyapatite-based bonemimicking scaffolds were used to recapitulate aspects of the in vivo tumour microenvironment, focusing on CSCs niche. The biological performance of human osteosarcoma cell lines (MG63 and SAOS-2) and enriched-CSCs were deeply analysed in these complex cell culture models. The results highlight the fundamental role of the tumour microenvironment proving the mimicry of osteosarcoma stem cell niche by the use of CSCs together with the biomimetic scaffolds, compared to conventional 2D culture systems. These advanced 3D cell culture in vitro tumour models could improve the predictivity of preclinical studies and strongly enhance the clinical translation.

Osteosarcoma is the most common primary malignant tumour of the bone ${ }^{1}$, frequently presenting in young people between the ages of 10-14 years and in adults over 65 years $^{2}$. Typically, patients with osteosarcoma are subjected to a combination of surgery, radiotherapy and chemotherapy ${ }^{3}$, which together can lead to a survival rate improvement of another 5 years in patients with localised tumour ${ }^{2,4-7}$. However, while overall survival rate of non-metastatic tumour is about $60-70 \%$, it remains less than $20 \%$ for patients with metastasis ${ }^{8}$. Moreover, tumour recurrences occur in $30-40 \%$ of non-metastatic patients ${ }^{9}$. Unfortunately, this scenario remained unchanged over the last 40 years, underlying the need of novel therapeutic strategies ${ }^{10,11}$. The therapeutic failure against osteosarcoma is mainly due to two reasons: (1) the lack of specificity for Cancer Stem Cells (CSCs) and (2) the absence of 3D microenvironment models that recapitulate the tumour complexity. Recent data have confirmed that osteosarcoma contains a distinct and defined cell population of CSCs, exhibiting stem-like phenotype with spherical colonies forming ability, called sarcospheres ${ }^{12}$, characterized by critical properties of invasiveness, migration and drug resistance ${ }^{13,14}$, suggesting their involvement in tumour progression, metastasis and recurrences frequently observed in osteosarcoma patients ${ }^{15}$. CSCs reside in an anatomically distinct and defined region inside the tumour microenvironment, called niche ${ }^{16,17}$, which controls CSC's fate through the mutual feedback between the different cells and the extracellular matrix $(\mathrm{ECM})^{18-21}$.

The second cause of the therapy failure is the lack of predictive in vitro models of the in vivo physio-pathological situation. Although many new tumour drug candidates seemed to be promising in in vitro screenings, they did not show any efficacy during in vivo studies ${ }^{22}$. This low translational ability can be attributed to the poor reliability of in vitro $2 \mathrm{D}$ standard models (i.e. plastic and glass surfaces) ${ }^{23}$. Although they are commonly used due to their controllability, simplicity, replicability and cheapness ${ }^{24,25}$, they do not reproduce the human disease complexity like the physico-chemical and mechanical tissue properties, the inter- and intra-tumour heterogeneity, the drug penetration through the tissue, the interaction between tumour cells and the stroma cells and the CSCs niche $1^{1,20,21,26,27}$. Recently, to overcome this great limitation, a significant effort has been made to clarify the dynamic interaction between the two players, cells and ECM, leading to the Tissue Engineering's evolution into "Tumour Engineering", with the aim to engineer the 3D tumour microenvironment and better elucidate several biological events and discover disruptive therapies ${ }^{1,28}$. 


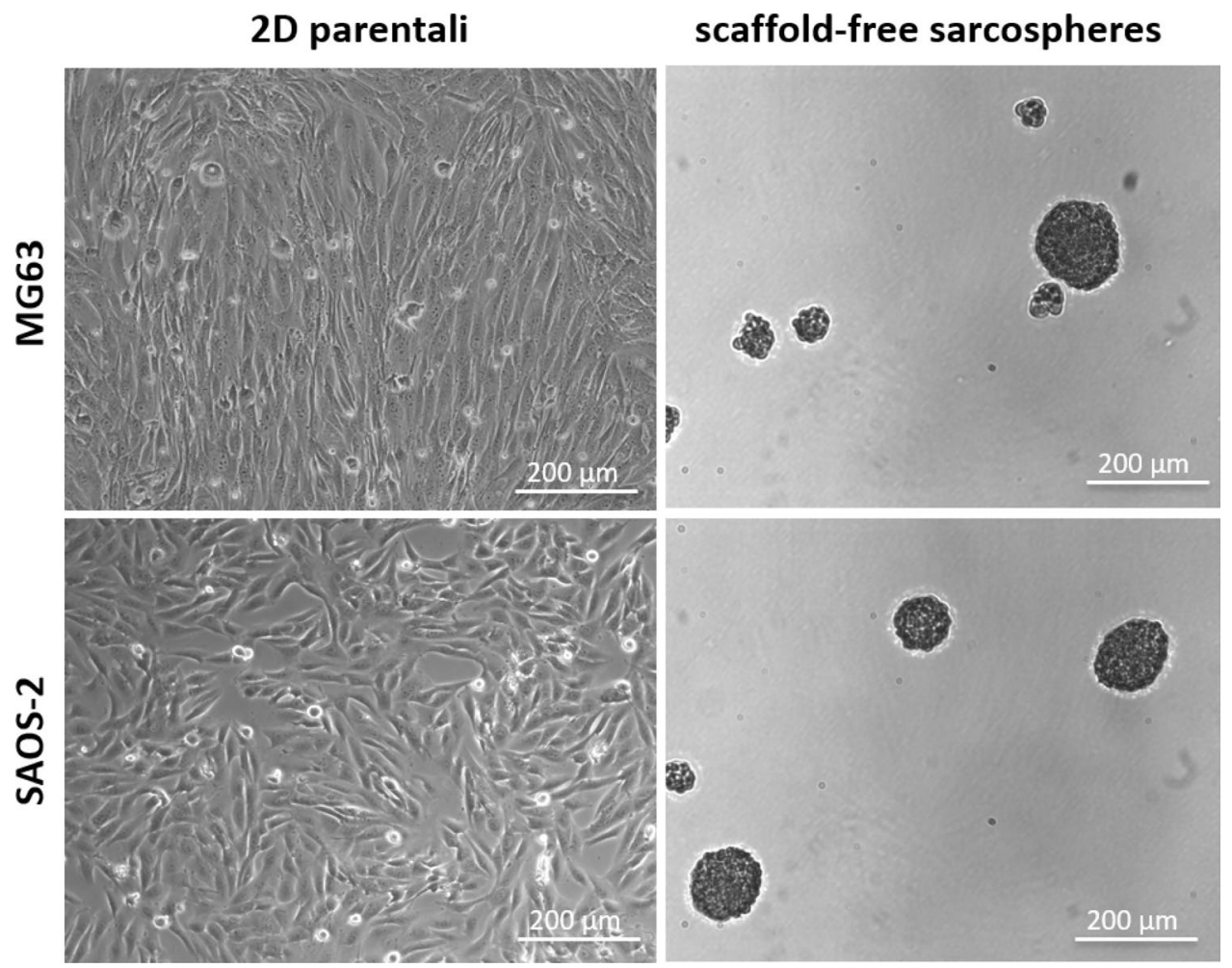

Figure 1. Qualitative morphological characterization of sarcospheres. Parental cells grown adherent to 2D standard support showing their typical morphologies. Stable floating sarcospheres.

In line with this challenge, we proposed two different 3D cancer models that recapitulate the native osteosarcoma heterogeneous CSCs niche. Our approach involved the combination of 3D scaffolds able to mimic the bone extracellular matrix, providing a structural support, specific physico-chemical and biomechanical stimuli to tumour cells ${ }^{29,30}$, together with enriched-CSCs obtained by sarcosphere-forming culture starting from osteosarcoma cell lines (MG63 and SAOS-2) ${ }^{31}$. In detail a biomimetic hybrid composite scaffolds obtained by a biomineralization process involving the direct nucleation of Mg-doped hydroxyapatite (MgHA) on self-assembling collagen fibres (MgHA/Coll) ${ }^{32,33}$ and porous hydroxyapatite scaffolds (HA) produced by direct foaming process $^{34}$, were used. An extensive characterization of morphology and gene expression profile of osteosarcoma cell lines and enriched-CSCs cultured in standard 2D conditions versus the proposed 3D culture systems has been performed. The results showed how the CSCs maintained more their stemness features when cultured in both MgHA/Coll and HA biomaterials compared to 2D model. The proposed biomimetic scaffolds, recapitulating the stem cell niche composition and architecture of native tissue, represent promising 3D tumour models that could replace the standard in vitro screening models closing the gap between the drug discovery and the clinical translation.

\section{Results}

Sarcospheres characterization. MG63 and SAOS-2 cell lines were subjected to sarcosphere-forming culture, following the well-established methods for CSCs enrichment ${ }^{35-37}$. After 10 days of culture, the scaffoldfree sarcospheres were characterized to confirm the successful CSCs' enrichment. A qualitative morphological evaluation confirmed the successful formation of stable CSCs' spheroids with diameter $\geq 50 \mu \mathrm{m}$ (Fig. 1).

The relative quantification of OCT-4, NANOG and SOX-2 genes was performed in order to define the increasing expression of stemness genes in the enriched-CSCs of the scaffold-free sarcospheres compared to 2D parental cells. The results showed a statistically significant higher expression of the three stemness markers in both cell lines. Transcriptional factor OCT-4, NANOG and SOX-2 were statistically significant higher in SAOS-2 sarcospheres ( $p$ value $\leq 0.0001$ for all genes) compared to 2D parental cells. MG63 sarcospheres showed a statistically significant higher expression of OCT-4 ( $p$ value $\leq 0.05)$, NANOG ( $p$ value $\leq 0.01)$ and SOX-2 ( $p$ value $\leq 0.001)$, compared to the parental control (Fig. 2).

In vitro 3D osteosarcoma models: analysis of cell-biomaterial interaction. Cell morphology analysis. After 10 days of culture, the cell-biomaterial interaction was analysed looking at the morphology of sarcospheres and the parental cells, respectively, grown in MgHA/Coll and HA 3D scaffolds. The H\&E staining of MgHA/Coll sample showed no differences between MG63 and SAOS-2 cells. In detail sarcospheres preserved 
a)

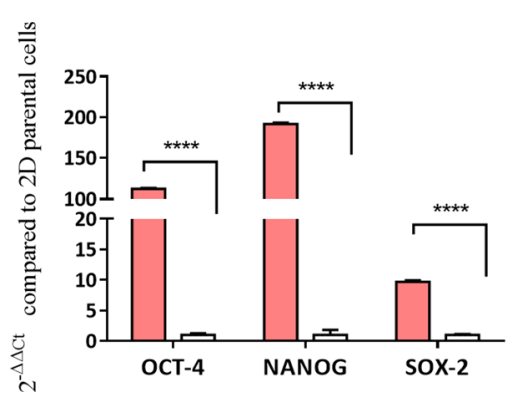

b)

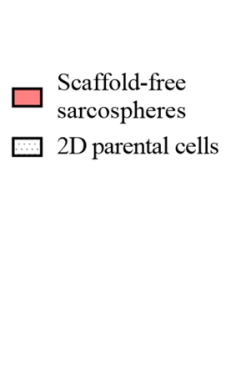

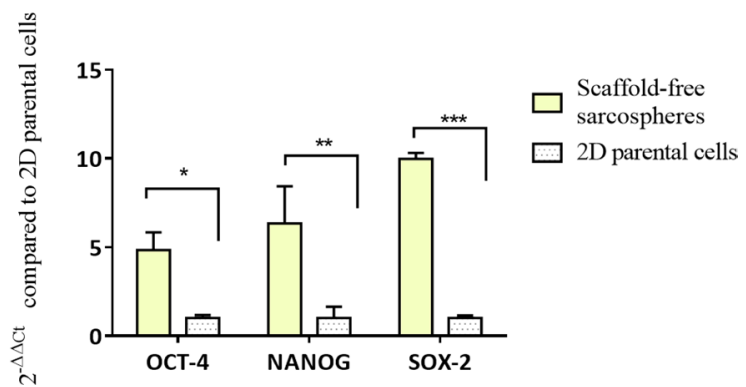

Figure 2. Gene expression analysis of scaffold-free sarcospheres. Relative quantification of scaffold-free sarcospheres gene expression of OCT-4, NANOG and SOX-2 in (a) SAOS-2 and (b) MG63 cell lines by qPCR. The graphs show the fold change expression of the genes relative to $2 \mathrm{D}$ parental cells (mean \pm standard error; ${ }^{* * * *} p$ value $\leq 0.0001 ;{ }^{* *} p$ value $\leq 0.001 ;{ }^{* *} p$ value $\leq 0.01 ;{ }^{*} p$ value $\left.\leq 0.05\right)$.

\section{D MgHA/Coll models}
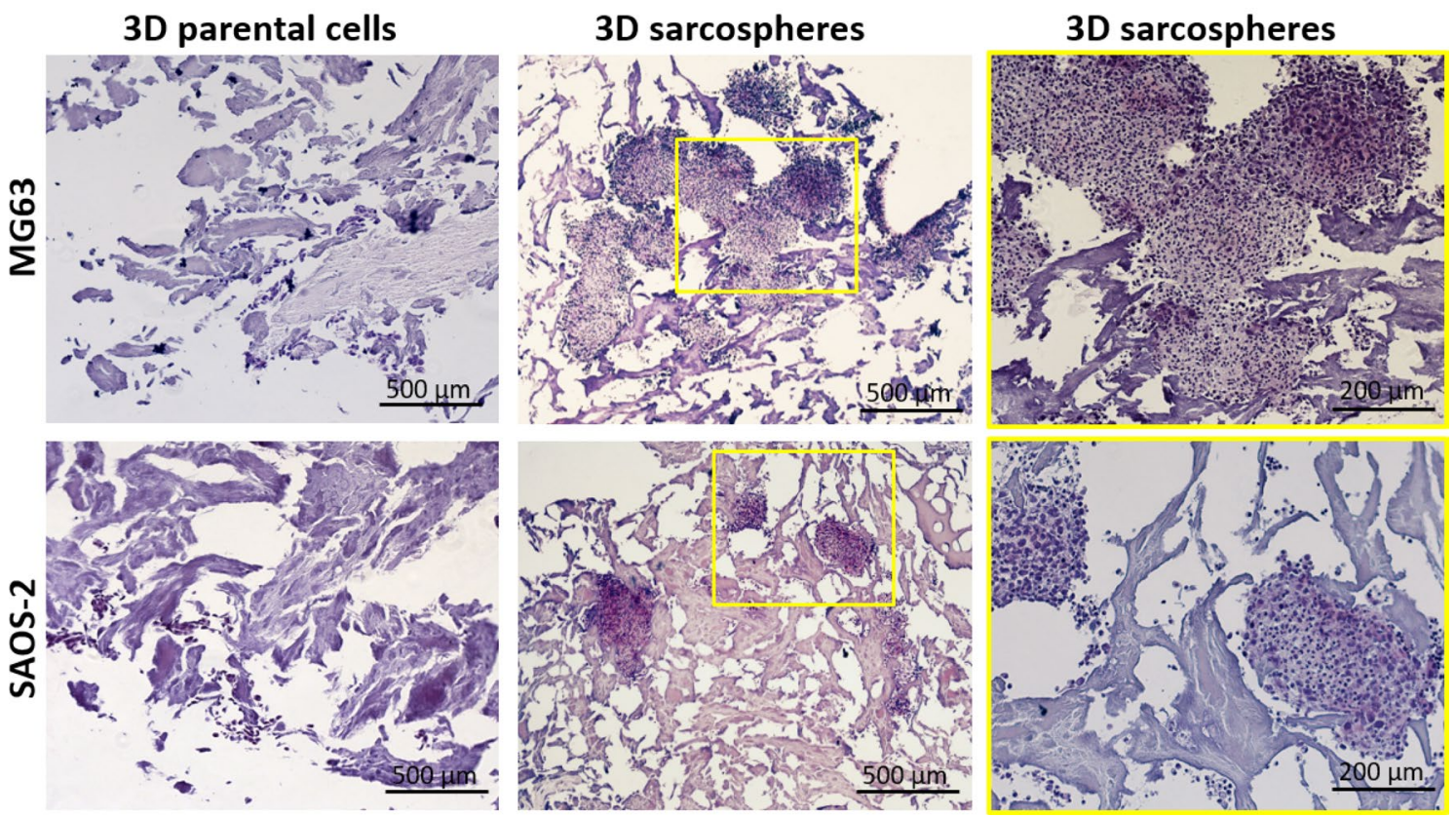

Figure 3. Histological analysis of the 3D MgHA/Coll scaffolds with both MG63 and SAOS-2 cells. The images

highlight the morphological features and the interaction behaviour of the sarcospheres and parental cells with the MgHA/Coll material. On the right, it is possible to observe an image enlargement of $200 \mu \mathrm{m}$ of the spheroidal phenotype of the sarcospheres.

their round-shape morphology, respect to parental cells, although they were well-embedded into the scaffold matrix (Fig. 3).

The actin filaments fluorescence analysis also confirmed an excellent cell-ECM interaction of both cell phenotypes with no significant differences. Figure 4 reported a representative panel of MG63 parental cells and MG63 sarcospheres grown in MgHA/Coll. The images highlight the complex interconnected structures of the scaffold and the maintenance of the cell-specific phenotypes morphology (Fig. 4).

The fluorescence analysis was performed also with the 3D HA model and it showed a peculiar round-shaped porous morphology of the HA scaffold (Fig. 5). The parental cells entirely colonized the biomaterial showing the typical adhesion morphology, while sarcospheres colonized the pores of the HA scaffolds preserving their spheroidal phenotype without differences between the MG63 and SAOS-2 (Fig. 5).

Likewise, SEM analysis confirmed the obtained results, providing a more detailed cell morphology evaluation of the 3D HA models (Fig. 6). Since no differences were observed in the morphology of the two cell lines, it has been reported a representative panel of SEM images of SAOS-2 cells. Parental cells were well spread over the biomaterial surface, with cytoplasmic extensions interconnecting cell-to-cell and cell-to-biomaterial nanostructured 


\section{D MgHA/Coll models}

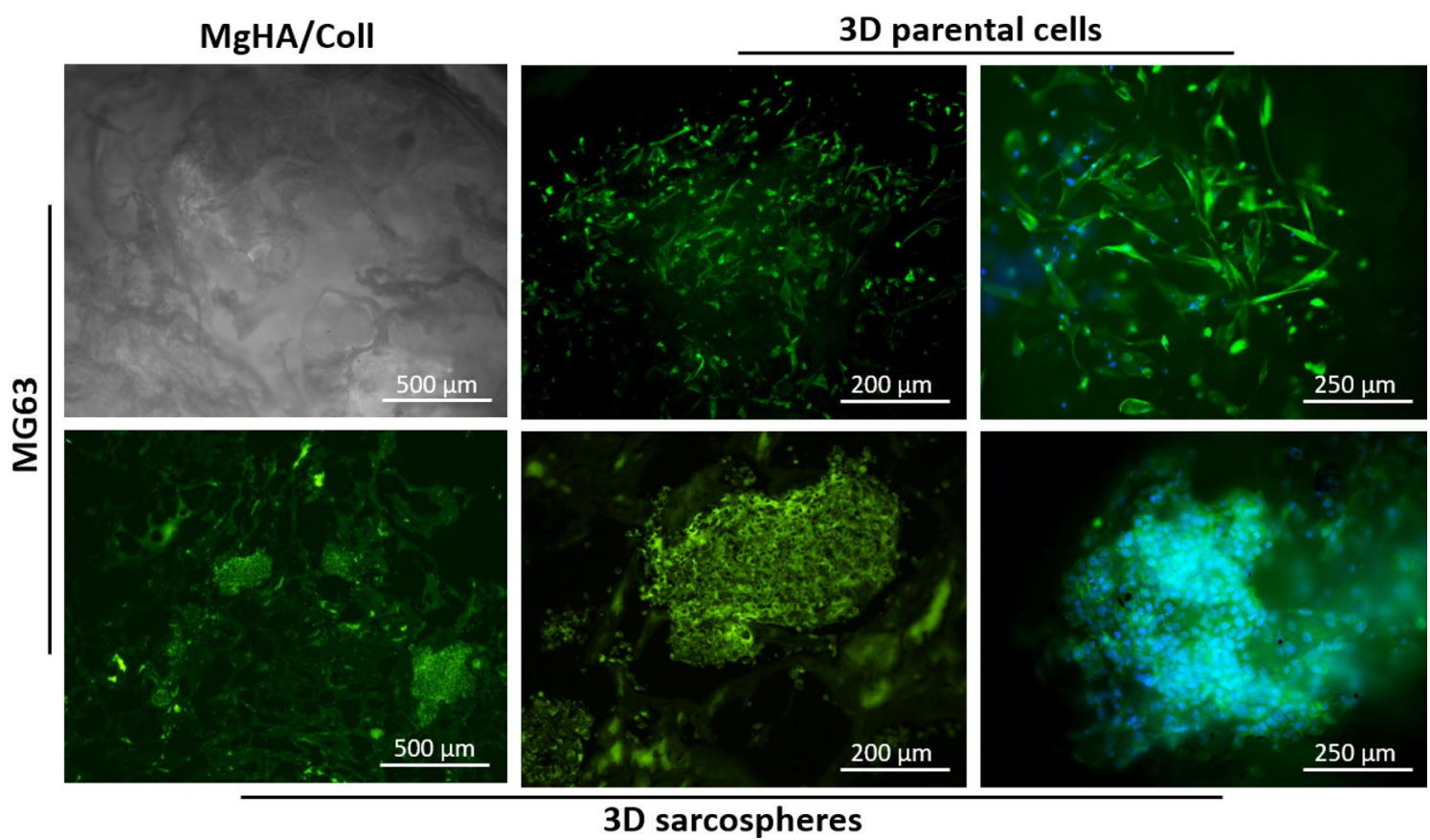

Figure 4. Fluorescence analysis of the 3D MgHA/Coll model of MG63 sarcospheres and parental cells. The top left figure is a representative image of the MgHA/Coll material. The panel shows the two cell phenotypes and their interaction with the hybrid material; cell nuclei in blue (DAPI) and F-actin filaments in green (Phalloidin).

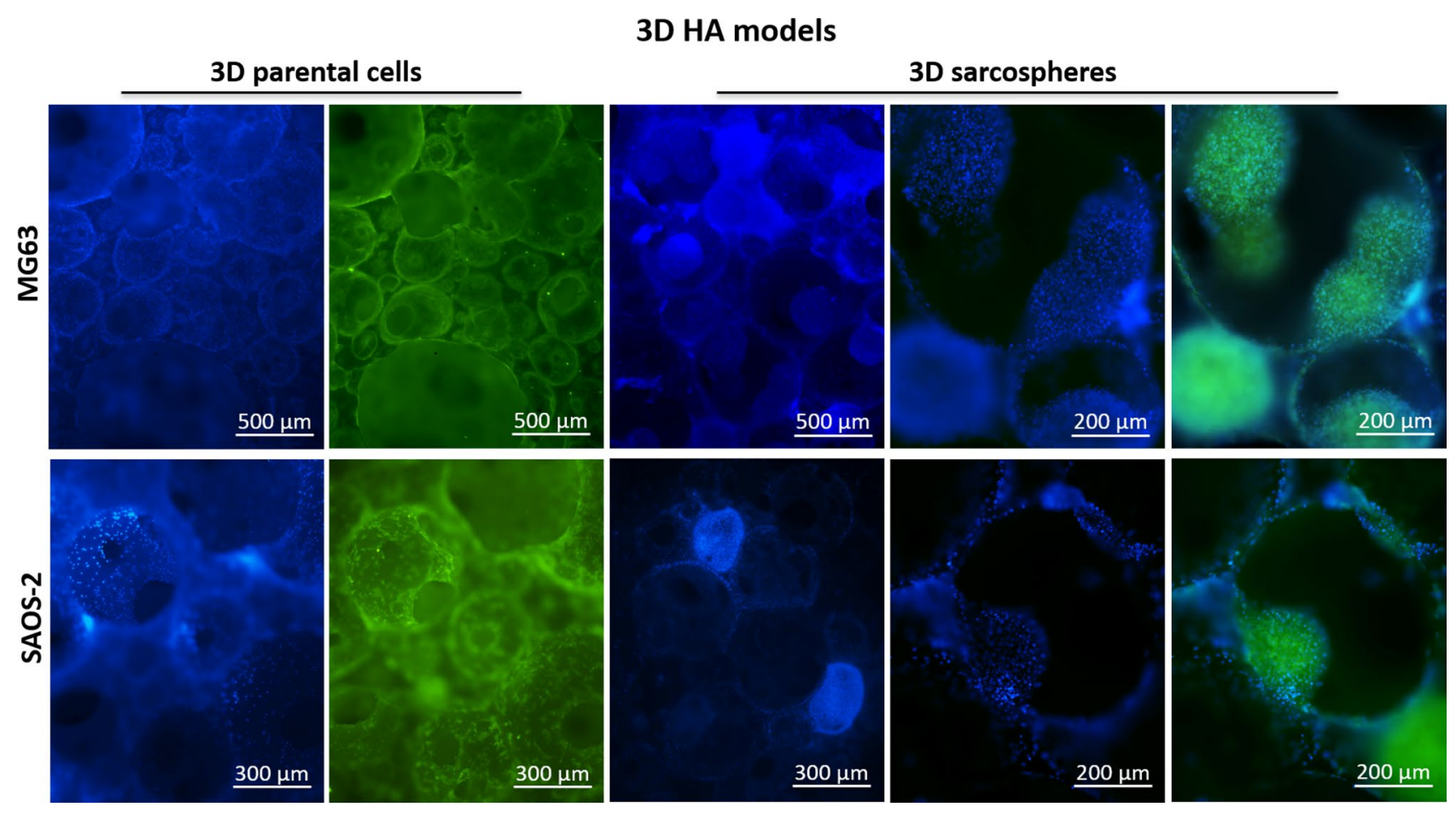

Figure 5. Fluorescence analysis of the 3D HA model of MG63 and SAOS-2 sarcospheres and parental cells. The images highlight the two cell phenotypes and their interaction with HA material; cell nuclei in blue (DAPI) and F-actin filaments in green (Phalloidin). 
3D HA models of SAOS-2
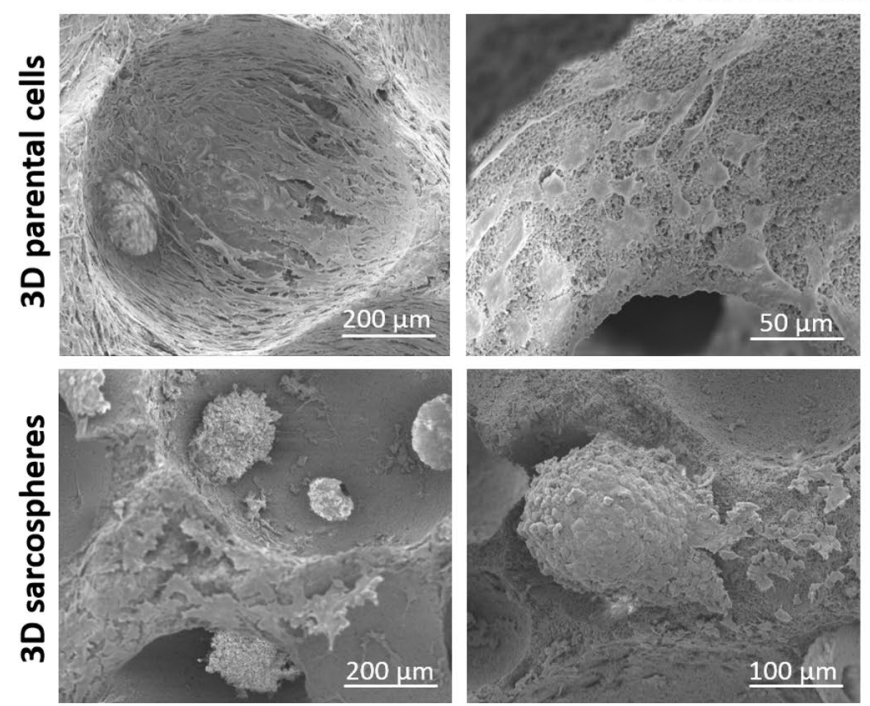
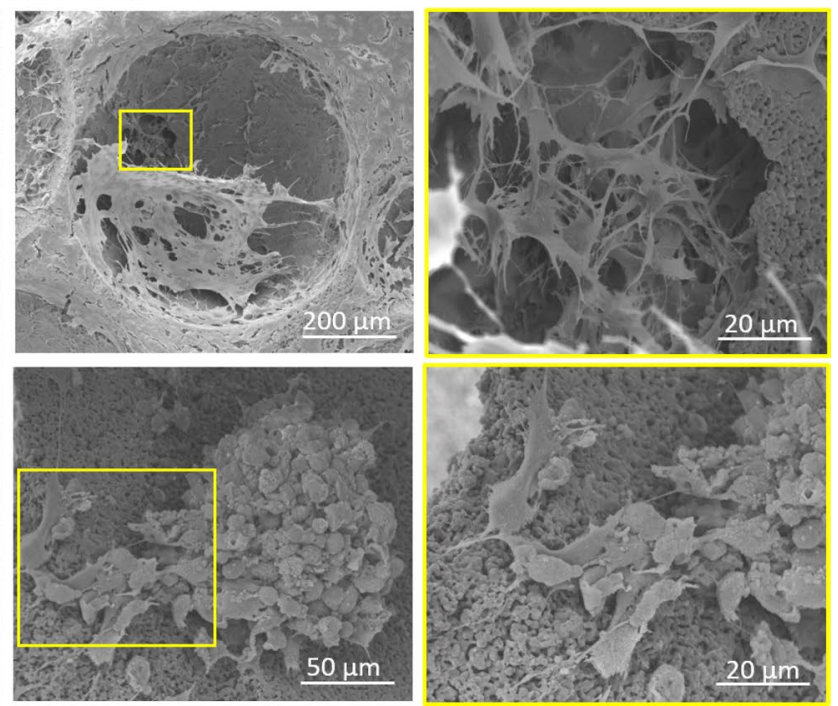

Figure 6. SEM analysis of the 3D HA model of SAOS-2 cells. The figure highlights phenotypic differences between sarcospheres and parental cells and interaction abilities of the cells toward HA scaffold.

surface. Moreover, the images showed the typical spheroidal phenotype of SAOS-2 sarcospheres, highlighting a tight sphere-edge interaction with the HA scaffold (Fig. 6).

Gene expression and immunofluorescence analysis. After 10 days of culture, the expression profile of genes involved in the stemness and CSCs/niche communication was relative quantified by qPCR (Fig. 7). OCT-4, NANOG and SOX-2 stemness marker genes were analysed to determine sarcospheres stem phenotype grown in 3D biomimetic scaffolds compared to the expression rate in the scaffold-free sarcospheres (Fig. 7a). The results showed that 3D sarcospheres scaffold-based models induced a significant up-regulation of NANOG in 3D HA model for SAOS-2 and MG63 cell line ( 40.9 and 4.8-fold change, respectively) ( $p$ value $\leq 0.0001$ and $\leq 0.05$, respectively) and in 3D MgHA/Coll model for MG63 cell line ( $\sim 4.6$-fold change) ( $p$ value $\leq 0.01$ ), compared to scaffold-free model. SAOS-2 sarcospheres also showed a significant increase of OCT-4 expression in both 3D HA ( 19.2-fold change) ( $p$ value $\leq 0.0001)$ and MgHA/Coll model ( 9.1-fold change) ( $p$ value $\leq 0.01)$. Finally, both cell lines showed a trend of increasing expression of SOX-2 in 3D scaffold-based models, even no statistically significant difference was detected (Fig. 7a).

Moreover, relative quantification of NOTCH-1, HIF- $1 \alpha$ and IL-6, the genes related to CSCs niche interaction, was carried out. The Fig. $7 \mathrm{~b}$ showed higher expression of signalling genes in the 3D scaffold-based sarcospheres compared to those without scaffolds. In detail, MG63 sarcospheres proved a significantly higher expression of NOTCH-1 ( 14.5-fold change) ( $p$ value $\leq 0.0001)$, and HIF- $1 \alpha(p$ value $\leq 0.001)$ when grown in both $3 \mathrm{D}$ $\mathrm{MgHA} / \mathrm{Coll}$ ( 4.0-fold change) and HA model ( 7.9-fold change), compared to scaffold-free sarcospheres. In the same way, SAOS-2 cell line showed a significant up-regulation of IL-6 in 3D sarcospheres with both material types ( 38.8-fold change and $\sim 40.6$-fold change in 3D HA and MgHA/Coll model, respectively) with a $p$ value $\leq 0.0001$ (Fig. 7b). The qualitative evaluation of OCT-4 and SOX-2, by immunofluorescence straining, confirmed the expression of these stemness markers. No differences have been observed in OCT- 4 and SOX-2 protein expression among the 3D scaffold-based models, therefore one representative image for each model has been reported (Fig. 7e,f,g,h).

\section{Discussion}

The incidence of osteosarcoma recurrence and metastasis due to the failure of the conventional therapies is strictly related to the critical role of CSCs, presented in several tumours, that exhibit stem-like phenotype and high drug resistance suggesting their involvement in tumour invasiveness and metastasis frequently observed in osteosarcoma patients ${ }^{15}$. Moreover, the lack of predictive in vitro models of the real physio-pathological scenario leads to a poor clinical translation of many apparently promising drugs candidates but also to fail in deciphering the biological events. In fact the 2D culture systems, used in preclinical drug-screening and in the study of cells and molecular behaviours, represent standard models but with plastic and/or glass surfaces very different from the osteosarcoma environment ${ }^{1,23,38,39}$.

The present study proposes a Tumour Engineering approach offering a radical change in the osteosarcoma in vitro studies through the development of advanced and alternative $3 \mathrm{D}$ cell culture in vitro models, able to mimic the in vivo tumour microenvironment. We studied the CSCs behaviour in two 3D biomimetic scaffolds ( $\mathrm{MgHA} / \mathrm{Coll}$ and $\mathrm{HA})$ and we proved how these bone-like biomaterials provide a more mimetic tumour microenvironment, in terms of nanostructure and physico-chemical features, compared to the $2 \mathrm{D}$ conventional culture systems. The MgHA/Coll and HA scaffolds were designed for bone regeneration and previously fully characterized by our group ${ }^{32-34,40-43}$. They are biocompatible, bioresorbable and they display osteogenic properties, 

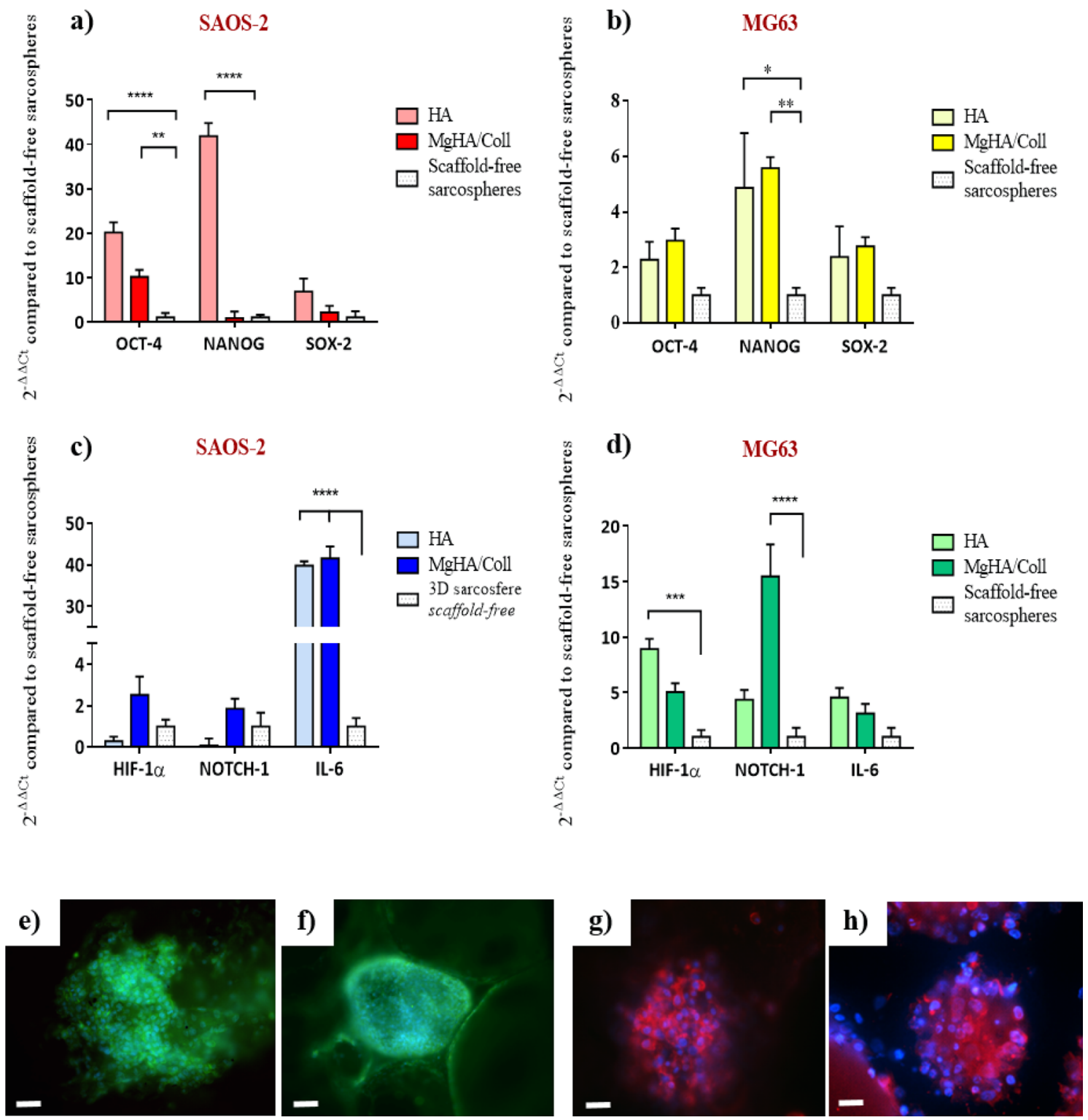

Figure 7. Gene expression and protein immunofluorescence analysis of 3D sarcospheres scaffold-based models. Relative quantification of OCT-4, NANOG and SOX-2, stemness marker genes, SAOS-2 (a) and MG63 (b). Relative quantification of HIF-1a, NOTCH-1 and IL-6, CSCs niche-related genes, SAOS-2 (c) and MG63 (d). The graphs show the fold change expression of the genes relative to the scaffold-free sarcospheres at day 10 of culture (mean \pm standard error; ${ }^{*} p$ value $\leq 0.05 ;{ }^{* *} p$ value $\leq 0.01 ;{ }^{* * *} p$ value $\leq 0.001 ;{ }^{* * *} p$ value $\leq 0.0001$;). The panel shows a representative image of OCT- 4 immunolocalization in SAOS-2 sarcorpheres MgHA/Coll model (e) and in HA model (f) scale bar $50 \mu \mathrm{m}$; SOX-2 immunolocalization in MG63 sarcorpheres MgHA/Coll model (g) and in HA model (h) scale bar $25 \mu \mathrm{m}$. Blu: cell nuclei; green: OCT-4; red: SOX-2.

mainly due to the presence of biomimetic $\mathrm{HA}^{44}$. The hybrid MgHA/Coll scaffold is obtained by a bio-inspired mineralization process reproducing the cascade of phenomena occurring in vivo during the formation of new bone tissue $\mathrm{e}^{32,33}$. In particular the process, carefully regulated by $\mathrm{pH}$ and temperature control, activates physicochemical and structural control mechanisms yielding the supramolecular assembling of Type I collagen fibrils and, at the same time, the heterogeneous nucleation of nanocrystals of $\mathrm{MgHA}$, so as to obtain a fibrous hybrid construct, stabilized by DHT crosslinking process, closely mimicking the woven bone tissue ${ }^{41,45,46}$ (Fig. 4). The HA scaffold is a sintered porous ceramic body obtained by a direct foaming process yielding open and highly interconnected macro/micro-porosity, thanks to the controlled formation of air bubbles incorporated into the hydroxyapatite ceramic slurry, obtained as the precursor of the final device $28,34,42,43,47-51$. Both the biomimetic scaffolds show a nanostructural organisation that, along with their peculiar chemistry, enhances the cell adhesion, migration and subsequent cell colonisation.

It has been well demonstrated that tumour spheroids provided high performance and a more accurate in vitro model for biological study of tumour and CSCs-tumour niche behaviour ${ }^{52}$, assuming the role of excellent candidates for the identification of novel therapeutic targets and the evaluation of sensitivity to chemotherapeutic 
agents in order to eradicate the CSCs niche ${ }^{53}$. With the aim to ascertain the efficiency of the CSCs enrichment method, both qualitative and quantitative analysis were performed to characterize the sarcospheres of both MG63 and SAOS-2 cell lines, by using the 2D parental cells model as experimental control. The results provide evidences of the formation of stable sarcospheres showing in vitro spheroidal phenotype and an higher mRNA level of stemness genes OCT-4, SOX-2 and NANOG ${ }^{36,54}$ compared to 2 D parental cells (Figs. 1, 2), confirming the successful CSCs enrichment by sarcospheres formation ${ }^{55,56}$. To achieve a more closed tumour biomimesis the sarcospheres were cultured in the 3D scaffolds. The morphological analysis confirmed the favourable environment for tumour cells. In fact the high biomimicry and bioactivity of both scaffolds did not induce the loss of the spheroidal phenotype of the sarcospheres, allowing their anchor within the HA pores and on MgHA/Coll fibres preserving their primary marker for CSCs identification ${ }^{57}$ without differences between the two cell lines (Figs. 3, 4, 5, 6).

It is well established that the presence of a bioactive $3 \mathrm{D}$ environment provides a higher stimulation of genes compared to a simple inducing medium, assuming high relevance for the reproduction of an in vitro more closely predictive osteosarcoma model ${ }^{58,59}$. In fact, many studies reported how the use of conventional 2D approaches failed to explain tumour cell biology, because they did not mimic real macrostructure, complexity (tumourstroma interactions) and heterogeneity of the tumour microenvironment ${ }^{28,60}$. In the proposed 3D models the significant upregulation of genes OCT-4, NANOG and SOX-2, typically used as stemness markers due to their essentiality in preserving the pluripotency and the self-renewal property of cancer stem cells ${ }^{36,61-64}$, demonstrated that the presence of 3D biomimetic scaffold, reproducing the nanostructure and physico-chemical features of native environment, induces the higher stem phenotype in 3D sarcospheres compared to those grown without scaffolds (Fig. 7a,b). The stemness was also confirmed by the detection of SOX-2 and OCT-4 proteins expressions, revealed by immunofluorescence analysis, in all the 3D scaffold-based models (Fig. 7e,f,g,h).

Moreover, it is well-known the significant contribute of ECM mechanical properties on tumour progression ${ }^{65}$, with particular attention to the stiffness of the material affecting tumour and stem cells fate and providing a tissue-specific microenvironment that plays a critical role in tumour development ${ }^{66,67}$. The two biomaterials used in this study exhibited significantly different, mechanical behaviours (i.e. Young's Modulus) giving the chance to compare the effect of high-stiffness HA scaffolds $(1.8 \pm 0.2 \mathrm{GPa})$ with low-stiffness MgHA/Coll scaffolds $(30.93 \pm 6.14 \mathrm{kPa})$ on sarcospheres behaviour ${ }^{32,34}$. Some studies reported that CSCs of osteosarcoma interacted optimally with $50-55 \mathrm{kPa}$ substrate stiffness ${ }^{68}$. In this respect, our results showed a higher expression of CSCs niche related-genes in 3D MgHA/Coll scaffold. In addition, it has been shown that in vitro invasive cancer cells have a higher affinity for matrices expressing the Type I Collagen ${ }^{69}$. At the same time, some studies reported that increasing ECM stiffness induces malignant phenotypes contributing to cancer progression and metastasis ${ }^{70,71}$, supporting the observed high expression levels of stemness genes in SAOS-2 sarcospheres in the 3D HA scaffold.

In order to clarify the role of the mimetic biomaterials on the CSCs niche-mediated stimuli, the expression of NOTCH-1, HIF-1 $\alpha$ and IL-6 was analysed. Although they have been observed different gene expression profiles related to the different scaffolds and cell types, overall a significant up-regulation of some of those genes was observed in 3D sarcospheres scaffold-based compared to those grown without scaffolds, confirming the greater mimesis of in vivo tumour microenvironment (Fig. 7c,d). In fact, these genes are typically involved in the signalling between CSCs and the tumour stem niche, developing a complex intercellular communication network regulating stemness and CSCs fate ${ }^{72}$. The signals are typically deregulated in osteosarcoma and they are involved in self-renewal, differentiation, drug resistance and metastatic potential regulation of CSCs ${ }^{73}$. In our study NOTCH-1 and HIF-1 $\alpha$ were higher expressed in MG63 sarcospheres grown in 3D MgHA/Coll and HA scaffold, respectively, compared to the scaffold-free model, confirming the active role of 3D mimetic environment on conservation of CSC phenotype ${ }^{74-76}$. Previous studies reported an increased level of IL-6 in the serum of patients with osteosarcoma ${ }^{77}$ and highlighted how IL-6, together with other cytokines (e.g. IL- $8^{78,79}$ and CXCL12 $2^{80}$ ), promotes immunosuppressive function, increasing chemoresistance ${ }^{81,82}$ and local and systemic tumour aggressiveness ${ }^{83}$. The IL-6 increase was observed also in our models where a statistically significant up-regulation in 3D sarcospheres of SAOS-2 cell line with both HA and MgHA/Coll biomaterial was reported (Fig. 7c).

Although the molecular analysis showed slight variances attributable to the intrinsic biological differences of the two osteosarcoma cell lines ${ }^{84}$ and to the specific biomimetic scaffold features ${ }^{49}$, the overall results confirmed that the use of the 3D scaffolds, together with sarcospheres implementation, improved the osteosarcoma stem cell niche microenvironment simulation, providing precise inputs supporting cell-cell and cell-ECM interactions and tumour signalling pathways in vitro. Also in vivo intratumoral variability can occur between tumour cells arising from the same mass, leading to the classification of different tumour cell subtypes that show a range of functional and morphological properties and a different molecular profile. Moreover, the extrinsic interactions between tumour cells and stromal microenvironments may be involved in the in vivo tumour heterogeneity as a crucial determinant of tumour malignancy ${ }^{84,85}$.

Although recognizing the limitations of our simplified 3D osteosarcoma models, they provided a more accurate starting point to understand the cellular and molecular mechanisms involved in cancer cells/biomatrix interactions, particularly in CSCs population. Most importantly, these models contribute to overcome the use of conventional 2D culture systems and to acquire overall awareness towards the implementation of $3 \mathrm{D}$ culture systems in our daily in vitro experiments and screenings.

\section{Methods}

Biomimetic scaffolds. The hybrid composite scaffold is composed of hydroxyapatite nanocrystals nucleated on self-assembled type I collagen fibres ( $\mathrm{MgHA} / \mathrm{Coll} \mathrm{60/40 \% )}$ and is obtained by a biomineralization process already reported in previous papers ${ }^{32,33,40}$. Briefly, an acid aqueous suspension was prepared by dispersing 
$150 \mathrm{~g}$ of $1 \mathrm{wt} . \%$ collagen gel (equine tendon derived type I collagen, $1 \mathrm{wt} . \%$ in aqueous acetic buffered solution $\mathrm{pH}$ 3.5, Opocrin SpA, Italy) into phosphoric acid solution (2.41 g in $500 \mathrm{ml} ; \mathrm{H}_{3} \mathrm{PO}_{4}, 85 \mathrm{wt} \%$, Sigma Aldrich, USA) at room temperature, while a basic aqueous suspension was obtained by adding $0.35 \mathrm{~g}$ of magnesium chloride $\left(\mathrm{MgCl}_{2} \cdot 6 \mathrm{H}_{2} \mathrm{O}, 99 \mathrm{wt}\right.$ \%, Sigma Aldrich, USA) into a calcium hydroxide suspension ( $2.71 \mathrm{~g}$ in $500 \mathrm{ml}$; $\mathrm{Ca}(\mathrm{OH})_{2}, 95 \mathrm{wt} . \%$, Sigma Aldrich, USA) at room temperature. Later, the acid suspension was dropped into the basic suspension at $25^{\circ} \mathrm{C}$ under constant stirring conditions causing the heterogeneous nucleation of MgHA nanocrystals onto the collagen fibres simultaneously at their assembling, thus forming a hybrid MgHA/Coll hydrogel. After $2 \mathrm{~h}$ of maturation at $25^{\circ} \mathrm{C}$, the hydrogel was lyophilized by freezing at $-40^{\circ} \mathrm{C}$ and drying at $25^{\circ} \mathrm{C}$ for $48 \mathrm{~h}$ under constant 0.086 mbar vacuum conditions (5 Pa, LIO $3000 \mathrm{PLT}$, Italy). Finally, the scaffolds were crosslinked by Dehydrothermal Treatment (DHT) into an oven at $160{ }^{\circ} \mathrm{C}$ for $48 \mathrm{~h}$ under a pressure of 0.01 $\operatorname{mbar}^{32,33}$.

The porous hydroxyapatite scaffold (HA) was obtained by a previously reported direct foaming process $^{34}$. Briefly, commercial HA powder (Riedel de Haen, Germany) was calcinated at $1000{ }^{\circ} \mathrm{C}$ for $5 \mathrm{~h}$ and dispersed in water with Dolapix CA (Zschimmer and Schwartz, Germany), following the weight ratio $\mathrm{HA}: \mathrm{H}_{2} \mathrm{O}$ :dispersant = 73:23:4. After $30 \mathrm{~min}$ stirring at $400 \mathrm{rpm}$ (Pulverisette 6, Fritsch, Germany) foaming agents were added to the suspension according to the HA powder amount, 2 wt.\% of Olympicon A (Olympia Surfactants, Italy) and $0.7 \mathrm{wt} . \%$ of W53 (Zschimmer and Schwartz, Germany), followed by further 5 min stirring. The asobtained foamed suspension was finally poured into paper moulds, dried for 2 days at room temperature and then sintered at $1250^{\circ} \mathrm{C}$ for 1 hour ${ }^{34}$.

Both types of biomaterial ( $3.00 \mathrm{~mm}$ high and $8.00 \mathrm{~mm}$ of diameter) were washed and sterilized by performing $25 \mathrm{kGy} \gamma$-ray irradiation before the use.

Cell culture. The Human Osteosarcoma SAOS-2 cell line purchased from American Type Culture Collection (ATCC hTB-85) were cultured in standard medium composed by McCoy's 5A (Modified) Medium (Gibco) supplemented with $15 \%$ of Foetal Bovine Serum (FBS) and 1\% of a mixture of Penicillin and Streptomycin (Pen/ Strep) $(100-100 \mu \mathrm{g} / \mathrm{ml})$.

The Human Osteosarcoma MG63 cell line purchased from American Type Culture Collection (ATCC CRL1427) were cultured in standard medium composed by Dulbecco's Modified Eagle Medium/F-12 Nutrient Mixture (DMEM/F-12) with Glutamine (GlutaMAX) (Gibco), supplemented with 10\% of FBS and 1\% of Pen/Strep. Both cell lines were kept in an incubator at $37^{\circ} \mathrm{C}$ and in $5 \% \mathrm{CO}_{2}$ atmosphere conditions. Cells were detached from culture plastics by trypsinization and centrifuged. The cell number and viability were determined with Trypan Blue Dye exclusion test. These cultures are named 2D parental cells models. All cell handling procedures were performed under laminar flow hood and in sterility conditions. The culture systems were incubated at $37^{\circ} \mathrm{C}$ and in $5 \% \mathrm{CO}_{2}$ atmosphere conditions.

Sarcosphere-forming culture. The sarcospheres were obtained from parental SAOS-2 and MG63 cell lines cultured under specific culture conditions as reported in literature ${ }^{35-37}$. Both cell lines were seeded in UltraLow Attachment 6 well-plates (Corning Inc., NY) with a density of 20,000 cells/well in DMEM/F-12 GlutaMAX (Gibco) culture medium supplemented with a specific factors cocktail composed by $10 \mu \mathrm{l} / \mathrm{ml} \mathrm{N} 2$ (Gibco), $20 \mu \mathrm{l} /$ $\mathrm{ml} \mathrm{B} 27$ (Gibco), $0.1 \mu \mathrm{l} / \mathrm{ml} \mathrm{FGF} \mathrm{(Invitrogen)} \mathrm{and} 0.01 \mu \mathrm{l} / \mathrm{ml}$ EGF (PeproTech). The cocktail was added to each well every $2 / 3$ days for a total of 10 days of culture at $37^{\circ} \mathrm{C}$ and in $5 \% \mathrm{CO}_{2}$ atmosphere conditions, observing sarcospheres formation. After their formation, the sarcospheres were mechanically dissociated by pipetting to facilitate cell counting and seeding in Ultra-Low Attachment 6-well plates with a density of $2.5 \times 10^{4}$ cells/well for additional 10 days of culture, following the manufacturer's instructions reported above. These new cultures were named sarcospheres scaffold-free models (Fig. 8a,b).

The 3D osteosarcoma models. The 3D scaffold-based culture models were developed for parental cells and sarcospheres of both cell lines (Fig. 8a). The scaffolds were placed in a 24-well plate and pre-conditioned in complete culture medium for $24 \mathrm{~h}$ before cell seeding. The scaffolds were seeded with parental cells or sarcospheres with a density of $2.5 \times 10^{4}$ cells/scaffold by carefully dropping $20 \mu \mathrm{L}$ of cell suspension on material upper surface. After $30 \mathrm{~min}$. incubation to allow cell pre-attachment, the specific culture media $(1.5 \mathrm{ml} /$ well $)$ for the different cell cultures, were added to each well. The standard culture medium of parental cells was gently changed every 3 days and sarcospheres were supplied with fresh culture medium and factors cocktail every 3 days for a total of 10 days of culture. These culture systems were named 3D sarcospheres or parental cells scaffold-based models (Fig. 8a,c).

Cell morphology analysis. Histological analysis. For histological analysis, the 3D MgHA/Coll models were washed in PBS $1 \mathrm{X}$ and fixed in $4 \%$ buffered formaldehyde for $15 \mathrm{~min}$ at room temperature. After washings in PBS 1X, the samples were placed in histological bio-cassettes and dehydrated with increasing scale alcohol passages (from 30 to $100 \% \mathrm{v} / \mathrm{v}$ ), under vacuum conditions. Then, the samples were subjected to two final dehydration passages in xylene for $1 \mathrm{~h}$ under the same conditions. Samples embedding was performed by using liquid paraffin. A semi-automatic rotary microtome (Histo-Line Laboratories) was used to dissect the samples obtaining polyline slides series of $5 \mu \mathrm{m}$ thick sections, which were hydrated with decreasing scale alcohol passages (from 100 to $30 \% \mathrm{v} / \mathrm{v}$ ) before Haematoxylin-Eosin (H\&E) staining. After staining, sections were mounted and visualized with an Optical Microscope (Nikon).

Actin and DAPI staining. The 3D MgHA/Coll and HA models were washed in PBS $1 \mathrm{X}$ (Gibco) for 5 min, then fixed in $4 \%(\mathrm{w} / \mathrm{v})$ paraformaldehyde (PFA) (Sigma) for $15 \mathrm{~min}$ and permeabilized in PBS $1 \mathrm{X}$ with $0.1 \%$ 
a)

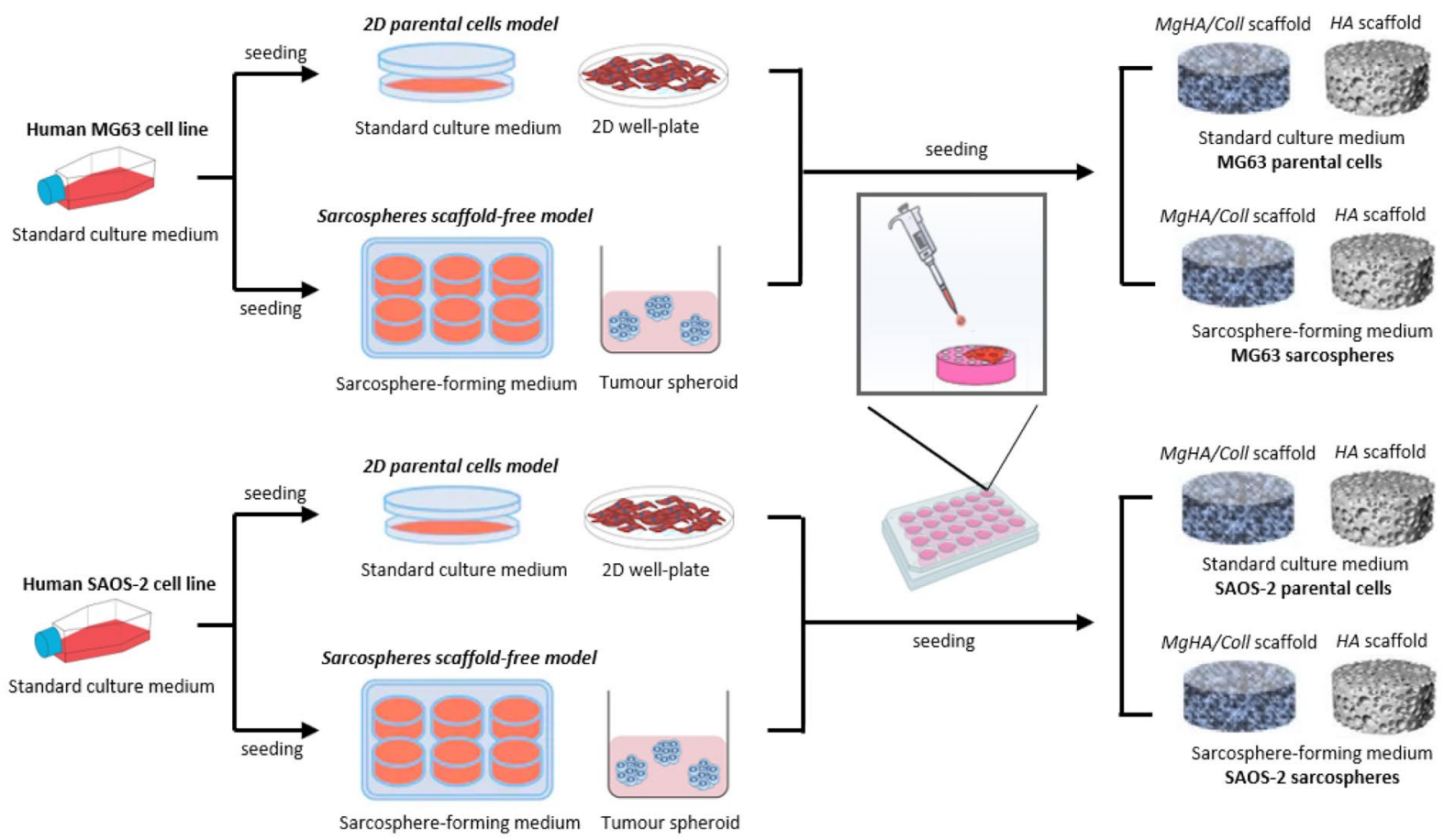

b) Sarcospheres characterization

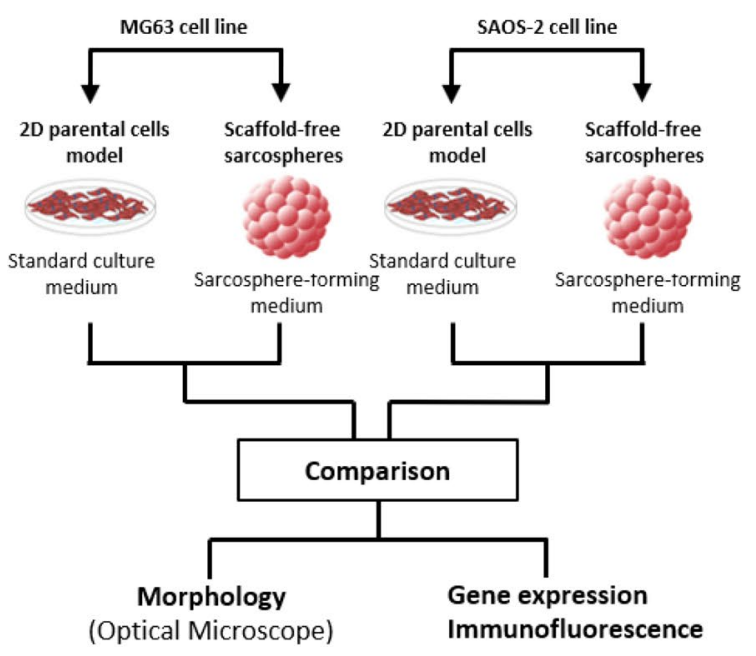

c)

3D bioscaffold-based model

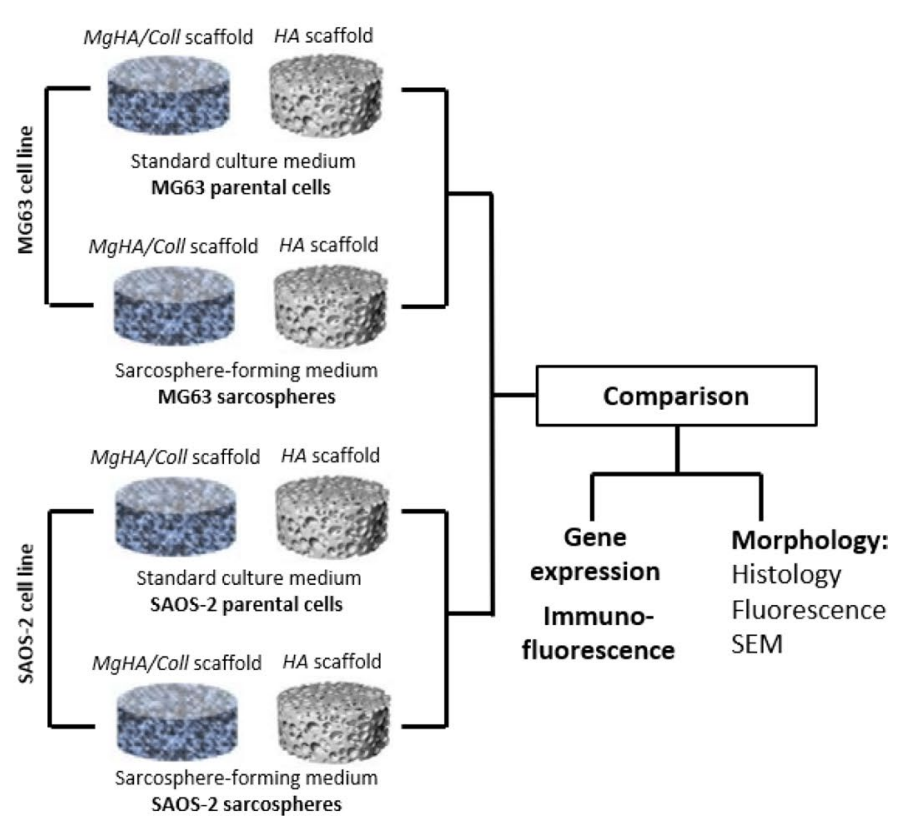

Figure 8. Graphical representation of the experimental plan. (a) Cell culture processing of tumour parental cells and sarcospheres of both cell lines for the development of the 3D scaffold-based models of osteosarcoma. (b) Sarcospheres characterization of both cell lines compared to 2D parental cells. (c) Experimental plan and characterization of the two phenotypes (parental and sarcospheres) in the 3D osteosarcoma models. Scaffoldfree sarcospheres were used as a control.

(v/v) Triton X-100 for $5 \mathrm{~min}$ at room temperature. F-actin filaments were highlighted with FITC-conjugated fluorescein-phalloidin (Invitrogen, $38 \mathrm{mM}$ ) staining, followed by 4'-6-Diamidino-2-phenylindole (DAPI) (Inv- 
itrogen, $300 \mu \mathrm{M}$ ) counterstaining to identify cell nuclei, following the manufacturer's instructions. The samples were visualized with an inverted Ti-E fluorescence microscope (Nikon).

Scanning electron microscopy (SEM) analysis. For SEM analysis, the 3D HA models were washed with $0.1 \mathrm{M}$ Sodium Cacodylate Buffer $\mathrm{pH} 7.4$ and fixed in 2.5\% Glutaraldehyde in $0.1 \mathrm{M}$ Sodium Cacodylate Buffer $\mathrm{pH}$ 7.4 for $2 \mathrm{~h}$ at $4{ }^{\circ} \mathrm{C}$. After washing in $0.1 \mathrm{M}$ Sodium Cacodylate Buffer $\mathrm{pH}$ 7.4, the samples were dehydrated with passages in a series of increasing scale alcohol, followed by two final passages with Hexamethyldisilazane (Sigma-Aldrich) at room temperature. Dehydrated samples were sputter-coated with gold (20 $\mu \mathrm{m}$ gold film) and observed by using Stereoscan 360 SEM (Cambridge Instruments, UK).

Quantitative real time PCR (qPCR). After 10 days of culture, the gene expression profile was analysed. The total RNA extraction and purification were performed by using the Tri Reagent and a purification kit (Directzol RNA MiniPrep kit, Zymo Research), following the manufacturer's instructions. RNA quantification and purity degree were evaluated by using the NanoDrop One Microvolume UV-Vis Spectrophotometer (Thermo Scientific), following the manufacturer's instructions. The single strand cDNA was produced by using the HighCapacity cDNA Reverse Transcription Kit (Applied Biosystem) starting from 500 ng of purified RNA, following the manufacturer's instructions. The cDNA was subjected to Real-Time PCR by using the TaqMan Gene Expression Assay Kit (Applied Biosystem) to SOX-2 (Hs01053049_s1), NOTCH (Hs01062014_m1), Inteleukin 6 (Hs00174131_m1) and HIF-1a (Hs00153153_m1) were analysed in the 3D sarcospheres scaffold-based models with both $\mathrm{HA}$ and MgHA/Coll material by using scaffold-free sarcospheres as experimental control. GAPDH (HS99999905-M1) was used as housekeeping gene. Two different experiments with the same experimental plan were performed and three samples of each group were analysed using three technical replicates. Data were collected from StepOne Real-Time PCR System (Applied Biosystems) and the relative quantification of target gene was performed by using the comparative threshold $(\mathrm{Ct})$ method $(\Delta \Delta \mathrm{Ct})$ where relative gene expression level equals to $2^{-\Delta \Delta \mathrm{Ct} 86}$.

Immunofluorescence analysis. The samples were fixed in $4 \%(\mathrm{w} / \mathrm{v})$ paraformaldehyde, saturated with $1 \%$ Bovine Serum Albumin (PAA The Cell Culture Company) and 10\% normal goat serum (Euroclone) for $30 \mathrm{~min}$ under slow agitation conditions. The samples were permeabilized with $0.3 \%$ (v/v) Triton X-100 for 20 min and incubated overnight at $4{ }^{\circ} \mathrm{C}$ with primary Anti-SOX-2 (1:200, MA1-014, Thermo Scientific), Anti-OCT-4/ POU5F1 ( $2 \mu \mathrm{g} / \mathrm{ml}$, Life Technologies). Secondary antibodies Alexa Fluor 488 goat anti-mouse (A11029, Molecular Probes) and Sheep Polyclonal anti-mouse (Ab50502, Abcam) were used for OCT-4 and SOX-2 detection, respectively, for $1 \mathrm{~h}$ in the dark at room temperature. Cell nuclei were stained with DAPI (300 nM). The images were acquired by an Inverted Ti-E fluorescence microscope (Nikon).

Statistical analysis. Statistical analysis was performed by using GraphPad Prism software (version 6.0). The results are expressed as mean \pm standard error of mean and they were analysed by two-way variance analysis (Two-way ANOVA) followed by Bonferroni’s multiple comparisons test.

Received: 11 June 2020; Accepted: 8 December 2020

Published online: 18 December 2020

\section{References}

1. De Luca, A. et al. Relevance of 3d culture systems to study osteosarcoma environment. J. Exp. Clin. Cancer Res. 37, 2 (2018).

2. Ottaviani, G. \& Jaffe, N. The epidemiology of osteosarcoma. Cancer Treat. Res. 152, 3-13 (2009).

3. Xiao, X. et al. HSP90AA1-mediated autophagy promotes drug resistance in osteosarcoma. J. Exp. Clin. Cancer Res. 37, 201 (2018).

4. Stiller, C. A. et al. Descriptive epidemiology of sarcomas in Europe: report from the RARECARE project. Eur. J. Cancer 49, 684-695 (2013).

5. Bacci, G. et al. Adjuvant and neoadjuvant chemotherapy for osteosarcoma of the extremities: 27 year experience at Rizzoli Institute, Italy. Eur. J. Cancer 41, 2836-2845 (2005).

6. DeLaney, T. F. et al. Radiotherapy for local control of osteosarcoma. Int. J. Radiat. Oncol. Biol. Phys. 61, 492-498 (2005).

7. Kager, L. et al. Osteosarcoma in very young children: experience of the Cooperative Osteosarcoma Study Group. Cancer 116, 5316-5324 (2010).

8. Gatta, G. et al. Childhood cancer survival trends in Europe: a EUROCARE Working Group study. J. Clin. Oncol. 23, 3742-3751 (2005).

9. Ferrari, S. et al. Postrelapse survival in osteosarcoma of the extremities: prognostic factors for long-term survival. J. Clin. Oncol. 21, 710-715 (2003).

10. Allison, D. C. et al. A meta-analysis of osteosarcoma outcomes in the modern medical era. Sarcoma 2012, 704872 (2012).

11. Roundhill, E. A., Jabri, S. \& Burchill, S. A. ABCG1 and Pgp identify drug resistant, self-renewing osteosarcoma cells. Cancer Lett. 453, 142-157 (2019).

12. Gibbs, C. P. et al. Stem-like cells in bone sarcomas: implications for tumorigenesis. Neoplasia 7, 967-976 (2005).

13. Kim, J. W., Tchernyshyov, I., Semenza, G. L. \& Dang, C. V. HIF-1-mediated expression of pyruvate dehydrogenase kinase: a metabolic switch required for cellular adaptation to hypoxia. Cell Metab. 3, 177-185 (2006).

14. Kuwai, T. et al. Expression of hypoxia-inducible factor-1alpha is associated with tumor vascularization in human colorectal carcinoma. Int. J. Cancer 105, 176-181 (2003).

15. Izadpanah, S. et al. Prospects for the involvement of cancer stem cells in the pathogenesis of osteosarcoma. J. Cell Physiol 235, 4167-4182 (2020).

16. Chen, S., Lee, B. H. \& Bae, Y. Notch signaling in skeletal stem cells. Calcif. Tissue Int. 94, 68-77 (2014).

17. Plaks, V., Kong, N. \& Werb, Z. The cancer stem cell niche: how essential is the niche in regulating stemness of tumor cells?. Cell Stem Cell 16, 225-238 (2015).

18. Fuchs, E., Tumbar, T. \& Guasch, G. Socializing with the neighbors: stem cells and their niche. Cell 116, 769-778 (2004).

19. Xie, T. \& Li, L. Stem cells and their niche: an inseparable relationship. Development 134, 2001-2006 (2007). 
20. Engler, A. J. et al. Myotubes differentiate optimally on substrates with tissue-like stiffness: pathological implications for soft or stiff microenvironments. J. Cell Biol. 166, 877-887 (2004).

21. Semler, E. J., Ranucci, C. S. \& Moghe, P. V. Mechanochemical manipulation of hepatocyte aggregation can selectively induce or repress liver-specific function. Biotechnol. Bioeng. 69, 359-369 (2000).

22. Toniatti, C., Jones, P., Graham, H., Pagliara, B. \& Draetta, G. Oncology drug discovery: planning a turnaround. Cancer Discov. 4, 397-404 (2014).

23. Horvath, P. et al. Screening out irrelevant cell-based models of disease. Nat. Rev. Drug Discov. 15, 751-769 (2016).

24. Edmondson, R., Broglie, J. J., Adcock, A. F. \& Yang, L. Three-dimensional cell culture systems and their applications in drug discovery and cell-based biosensors. Assay Drug Dev. Technol. 12, 207-218 (2014).

25. Huh, D., Hamilton, G. A. \& Ingber, D. E. From 3D cell culture to organs-on-chips. Trends Cell Biol. 21, 745-754 (2011).

26. Discher, D. E., Janmey, P. \& Wang, Y. L. Tissue cells feel and respond to the stiffness of their substrate. Science 310, 1139-1143 (2005).

27. Scheideler, L., Rupp, F., Wendel, H. P., Sathe, S. \& Geis-Gerstorfer, J. Photocoupling of fibronectin to titanium surfaces influences keratinocyte adhesion, pellicle formation and thrombogenicity. Dent. Mater. 23, 469-478 (2007).

28. Fong, E. L., Harrington, D. A., Farach-Carson, M. C. \& Yu, H. Heralding a new paradigm in 3D tumor modeling. Biomaterials 108, 197-213 (2016).

29. Lu, W. D. et al. Development of an acellular tumor extracellular matrix as a three-dimensional scaffold for tumor engineering. PLOS ONE 9, e103672 (2014).

30. Hutmacher, D. W. et al. Can tissue engineering concepts advance tumor biology research?. Trends Biotechnol. 28, 125-133 (2010).

31. Kamal, A. F. et al. Isolation, Culture and Characterization of Cancer Stem Cells from Primary Osteosarcoma. Open Stem Cell J. 5, $1-13(2018)$.

32. Krishnakumar, G. S. et al. Evaluation of different crosslinking agents on hybrid biomimetic collagen-hydroxyapatite composites for regenerative medicine. Int. J. Biol. Macromol. 106, 739-748 (2018).

33. Tampieri, A. et al. Biologically inspired synthesis of bone-like composite: self-assembled collagen fibers/hydroxyapatite nanocrystals. J. Biomed. Mater. Res. A 67, 618-625 (2003).

34. Dapporto, M., Fabbic, C., Figallo, E. \& Tampieri, A. A novel route for the synthesis of macroporous bioceramics for boneregeneration. J. Eur. Ceramic Soc. 36, 2383-2388 (2016).

35. Brown, H. K., Tellez-Gabriel, M. \& Heymann, D. Cancer stem cells in osteosarcoma. Cancer Lett. 386, 189-195 (2017).

36. Lee, Y. H. et al. DHFR and MDR1 upregulation is associated with chemoresistance in osteosarcoma stem-like cells. Oncol. Lett. 14, 171-179 (2017).

37. Tang, Q. L. et al. Salinomycin inhibits osteosarcoma by targeting its tumor stem cells. Cancer Lett. 311, 113-121 (2011).

38. Pampaloni, F., Reynaud, E. G. \& Stelzer, E. H. The third dimension bridges the gap between cell culture and live tissue. Nat. Rev. Mol. Cell Biol. 8, 839-845 (2007).

39. Achilli, T. M., Meyer, J. \& Morgan, J. R. Advances in the formation, use and understanding of multi-cellular spheroids. Expert Opin. Biol. Ther. 12, 1347-1360 (2012).

40. Krishnakumar, G. S. et al. Ribose mediated crosslinking of collagen-hydroxyapatite hybrid scaffolds for bone tissue regeneration using biomimetic strategies. Mater. Sci. Eng. C Mater. Biol. Appl. 77, 594-605 (2017).

41. Tampieri, A., Sprio, S., Sandri, M. \& Valentini, F. Mimicking natural bio-mineralization processes: a new tool for osteochondral scaffold development. Trends Biotechnol. 29, 526-535 (2011).

42. Panseri, S. et al. Magnetic hydroxyapatite bone substitutes to enhance tissue regeneration: evaluation in vitro using osteoblast-like cells and in vivo in a bone defect. PLoS ONE 7, e38710 (2012).

43. Scaglione, S. et al. Order versus disorder: in vivo bone formation within osteoconductive scaffolds. Sci. Rep. 2, 274 (2012).

44. Montesi, M., Panseri, S., Iafisco, M., Adamiano, A. \& Tampieri, A. Effect of hydroxyapatite nanocrystals functionalized with lactoferrin in osteogenic differentiation of mesenchymal stem cells. J. Biomed. Mater. Res. A 103, 224-234 (2015).

45. Tampieri, A. et al. Design of graded biomimetic osteochondral composite scaffolds. Biomaterials 29, 3539-3546 (2008).

46. Panseri, S. et al. Biomimetic scaffold with aligned microporosity designed for dentin regeneration. Front. Bioeng. Biotechnol. 4, 48 (2016).

47. Sionkowska, A. \& Kozlowska, J. Characterization of collagen/hydroxyapatite composite sponges as a potential bone substitute. Int. J. Biol. Macromol. 47, 483-487 (2010).

48. Tampieri, A. et al. Heterogeneous chemistry in the 3-D state: an original approach to generate bioactive, mechanically-competent bone scaffolds. Biomater. Sci. 7, 307-321 (2018).

49. Deans, T. L., Singh, A., Gibson, M. \& Elisseeff, J. H. Regulating synthetic gene networks in 3D materials. Proc. Natl. Acad. Sci. U. S. A. 109, 15217-15222 (2012).

50. Salamanna, F., Contartese, D., Maglio, M. \& Fini, M. A systematic review on in vitro 3D bone metastases models: a new horizon to recapitulate the native clinical scenario?. Oncotarget 7, 44803-44820 (2016).

51. Brigo, L. et al. 3D high-resolution two-photon crosslinked hydrogel structures for biological studies. Acta Biomater. 55, 373-384 (2017).

52. Ivanov, D. P. \& Grabowska, A. M. Spheroid arrays for high-throughput single-cell analysis of spatial patterns and biomarker expression in 3D. Sci. Rep. 7, 41160 (2017).

53. Guo, X. et al. miR-335 negatively regulates osteosarcoma stem cell-like properties by targeting POU5F1. Cancer Cell Int. 17, 29 (2017).

54. Zhang, C., Ma, K. \& Li, W. Y. IL-6 promotes cancer stemness and oncogenicity in U2OS and MG-63 osteosarcoma cells by upregulating the OPN-STAT3 pathway. J. Cancer 10, 6511-6525 (2019).

55. Huang, K. et al. Size-dependent localization and penetration of ultrasmall gold nanoparticles in cancer cells, multicellular spheroids, and tumors in vivo. ACS Nano 6, 4483-4493 (2012).

56. Dilnawaz, F. \& Sahoo, S. K. Enhanced accumulation of curcumin and temozolomide loaded magnetic nanoparticles executes profound cytotoxic effect in glioblastoma spheroid model. Eur. J. Pharm. Biopharm. 85, 452-462 (2013).

57. Bouchet, B. P. \& Akhmanova, A. Microtubules in 3D cell motility. J. Cell Sci. 130, 39-50 (2017).

58. Kundu, B. et al. Mechanical property of hydrogels and the presence of adipose stem cells in tumor stroma affect spheroid formation in the 3D osteosarcoma model. ACS Appl. Mater. Interfaces 11, 14548-14559 (2019).

59. Monteiro, M. V., Gaspar, V. M. Ferreira, L. P. \& Mano, J. F. Hydrogel 3D in vitro tumor models for screening cell aggregation mediated drug response. Biomater. Sci. 8, 1855-1864 (2020).

60. Charoen, K. M., Fallica, B., Colson, Y. L., Zaman, M. H. \& Grinstaff, M. W. Embedded multicellular spheroids as a biomimetic 3D cancer model for evaluating drug and drug-device combinations. Biomaterials 35, 2264-2271 (2014).

61. Lu, Y. et al. Knockdown of Oct4 and Nanog expression inhibits the stemness of pancreatic cancer cells. Cancer Lett. 340, 113-123 (2013).

62. Iv Santaliz-Ruiz, L. E., Xie, X., Old, M., Teknos, T. N. \& Pan, Q. Emerging role of nanog in tumorigenesis and cancer stem cells. Int. J. Cancer 135, 2741-2748 (2014).

63. Huang, Y., Dai, H. \& Guo, Q. N. TSSC3 overexpression reduces stemness and induces apoptosis of osteosarcoma tumor-initiating cells. Apoptosis 17, 749-761 (2012).

64. Basu-Roy, U. et al. Sox2 maintains self renewal of tumor-initiating cells in osteosarcomas. Oncogene 31, 2270-2282 (2012). 
65. Cox, T. R. \& Erler, J. T. Remodeling and homeostasis of the extracellular matrix: implications for fibrotic diseases and cancer. Dis. Model. Mech. 4, 165-178 (2011).

66. Engler, A. J., Sen, S., Sweeney, H. L. \& Discher, D. E. Matrix elasticity directs stem cell lineage specification. Cell 126, 677-689 (2006).

67. Friedl, P. \& Wolf, K. Tube travel: the role of proteases in individual and collective cancer cell invasion. Cancer Res. 68, 7247-7249 (2008).

68. Jabbari, E., Sarvestani, S. K., Daneshian, L. \& Moeinzadeh, S. Optimum 3D matrix stiffness for maintenance of cancer stem cells is dependent on tissue origin of cancer cells. PLoS ONE 10, e0132377 (2015).

69. Taubenberger, A. V. et al. 3D extracellular matrix interactions modulate tumour cell growth, invasion and angiogenesis in engineered tumour microenvironments. Acta Biomater. 36, 73-85 (2016).

70. Chaudhuri, O. et al. Extracellular matrix stiffness and composition jointly regulate the induction of malignant phenotypes in mammary epithelium. Nat. Mater. 13, 970-978 (2014).

71. Acerbi, I. et al. Human breast cancer invasion and aggression correlates with ECM stiffening and immune cell infiltration. Integr. Biol. (Camb.) 7, 1120-1134 (2015).

72. Ginestier, C. et al. CXCR1 blockade selectively targets human breast cancer stem cells in vitro and in xenografts. J. Clin. Investig. 120, 485-497 (2010)

73. Jinushi, M., Baghdadi, M., Chiba, S. \& Yoshiyama, H. Regulation of cancer stem cell activities by tumor-associated macrophages. Am. J. Cancer Res. 2, 529-539 (2012).

74. McQueen, P. et al. The Wnt signaling pathway: implications for therapy in osteosarcoma. Expert Rev. Anticancer Ther. 11, 1223-1232 (2011).

75. Alfranca, A. et al. Bone microenvironment signals in osteosarcoma development. Cell Mol. Life Sci. 72, 3097-3113 (2015).

76. Guo, M. et al. Hypoxia promotes migration and induces CXCR4 expression via HIF-1a activation in human osteosarcoma. PLoS ONE 9, e90518 (2014).

77. Xiao, H. et al. Effect of the cytokine levels in serum on osteosarcoma. Tumour Biol. 35, 1023-1028 (2014).

78. Du, L. et al. CXCR1/Akt signaling activation induced by mesenchymal stem cell-derived IL-8 promotes osteosarcoma cell anoikis resistance and pulmonary metastasis. Cell Death Dis. 9, 714 (2018).

79. Vernot, J. P. Senescence-associated pro-inflammatory cytokines and tumor cell plasticity. Front. Mol. Biosci. 7, 63 (2020).

80. Mortezaee, K. CXCL12/CXCR4 axis in the microenvironment of solid tumors: a critical mediator of metastasis. Life Sci. 249, $117534(2020)$

81. Tu, B. et al. Mesenchymal stem cells promote osteosarcoma cell survival and drug resistance through activation of STAT3. Oncotarget 7, 48296-48308 (2016)

82. Duan, Z., Lamendola, D. E., Penson, R. T., Kronish, K. M. \& Seiden, M. V. Overexpression of IL-6 but not IL-8 increases paclitaxel resistance of U-2OS human osteosarcoma cells. Cytokine 17, 234-242 (2002).

83. Avnet, S. et al. Increased osteoclast activity is associated with aggressiveness of osteosarcoma. Int. J. Oncol. 33, 1231-1238 (2008).

84. Visvader, J. E. Cells of origin in cancer. Nature 469, 314-322 (2011).

85. Tlsty, T. D. \& Coussens, L. M. Tumor stroma and regulation of cancer development. Annu. Rev. Pathol. 1, 119-150 (2006).

86. Livak, K. J. \& Schmittgen, T. D. Analysis of relative gene expression data using real-time quantitative PCR and the 2(-Delta Delta C(T)) Method. Methods 25, 402-408 (2001).

\section{Author contributions}

M.M. and S.P. conceived and designed the experiments. G.B., M.M., S.P. conducted the experiments. G.B., M.M., S.P. analysed the results. E.C. and M.D., synthesized the biomaterials. M.S. and S.S. supervised the biomaterials synthesis. G.B., M.M., S.P. wrote the paper. All authors reviewed the manuscript.

\section{Competing interests}

The authors declare no competing interests.

\section{Additional information}

Correspondence and requests for materials should be addressed to G.B. or M.M.

Reprints and permissions information is available at www.nature.com/reprints.

Publisher's note Springer Nature remains neutral with regard to jurisdictional claims in published maps and institutional affiliations.

Open Access This article is licensed under a Creative Commons Attribution 4.0 International License, which permits use, sharing, adaptation, distribution and reproduction in any medium or format, as long as you give appropriate credit to the original author(s) and the source, provide a link to the Creative Commons licence, and indicate if changes were made. The images or other third party material in this article are included in the article's Creative Commons licence, unless indicated otherwise in a credit line to the material. If material is not included in the article's Creative Commons licence and your intended use is not permitted by statutory regulation or exceeds the permitted use, you will need to obtain permission directly from the copyright holder. To view a copy of this licence, visit http://creativecommons.org/licenses/by/4.0/.

(C) The Author(s) 2020 